\title{
A case-control study of lifestyle risk factors associated with tuberculosis in Liverpool, North-West England
}

\author{
K. Tocque*, M.A. Bellis*, N.J. Beeching", Q. Syed", T. Remmington", P.D.O. Davies ${ }^{+}$
}

A case-control study of lifestyle risk factors associated with tuberculosis in Liverpool, North-West England. K. Tocque, M.A. Bellis, N.J. Beeching, Q. Syed, T. Remmington, P.D.O. Davies. (C) ERS Journals Ltd 2001.

ABSTRACT: The aim of this study was to identify the subtle influences of exposure and individual lifestyles on the risk of developing tuberculosis.

A retrospective case-control study (with matching by sex, age, postcode and ethnicity) of all tuberculosis cases notified over a 7-yr period in Liverpool, UK, was carried out.

Multiple logistic regression showed that, before diagnosis, cases were 7.4 times more likely to have had visitors from abroad; 4.0 times more likely to have been born abroad; and 3.8 times more likely to have lived with someone with tuberculosis. Subtle socioeconomic factors were also evident with cases 4.0 times less likely to have additional bathrooms. Lifestyle factors emerged with cases 2.3 times more likely to have smoked for at least $\mathbf{3 0} \mathrm{yrs}, \mathbf{3 . 8}$ times less likely to eat dairy products every week and 2.6 times less likely to have had high blood pressure. At interview, these factors were still evident, but cases, unlike controls, had reduced their smoking and alcohol consumption and were less likely to go out of the home or exercise than before their illness.

Within individuals, lifestyle consequences of tuberculosis lead to a "healthier" lifestyle on the one hand (less smoking and alcohol consumption), but a reduced quality of life (social activity) on the other.

Eur Respir J 2001; 18: 959-964.
* North West Public Health Observatory, John Moores University, ${ }^{\#}$ Liverpool School of Tropical Medicine, Communicable Disease Surveillance Centre (North West), ${ }^{+}$Tuberculosis Research Unit, Liverpool, UK

Correspondence: K. Tocque, North West Public Health Observatory, Public Health Sector, Liverpool John Moores University, 70 Great Crosshall Street, Liverpool, L3 2AB, UK. Fax: 4401512314435

Keywords: Disease, exercise, exposure, quality of life, smoking, socioeconomic

Received: February 42001

Accepted after revision July 312001

The study was funded by the Smith Charity, and was approved by the Research Ethics Committees of Aintree Hospitals NHS Trust, Cardiothoracic Centre Broadgreen Hospital and South Sefton Health Authority.
One-third of the global population is infected with Mycobacterium tuberculosis and it is estimated that 30 million people will die from tuberculosis during the next $10 \mathrm{yrs}$ [1]. The recent resurgence of tuberculosis in England and Wales is estimated to have resulted in 8,000 more cases of tuberculosis, between 1988-1993, than would have been predicted from previous trends [2].

Within the developing world, the reasons proposed for the increase in disease are population expansion, inadequate coverage by control programmes, poor cure rates and case holding and the interaction of human immunodeficiency virus (HIV) infection with tuberculosis [3, 4]. Within the developed world, the recent increase has principally been associated with immigration from developing countries [5-7], increasing poverty [8-10], unemployment [10], overcrowding [11], smoking [12], homelessness [13, 14], an ageing indigenous population $[15,16]$ and, particularly within the USA, HIV infection [17, 18]. However, other studies, by analysing several geographical measures together, have shown that rates of disease across urban areas of England were geographically more closely associated with the proportion of the population that were of non-White ethnic origin than with economic deprivation indices [19].

The majority of individuals in a population do not develop tuberculosis, either due to lack of exposure or due to individual characteristics that limit development of the disease after exposure [20]. The city of Liverpool has experienced one of the most rapid increases in tuberculosis cases in the UK, with a $30 \%$ rise between 1987-1993. Given this rise in tuberculosis and the known link between social deprivation and rates of disease within electoral wards in the city [8], the authors have used Liverpool as a model to test the hypothesis that the exposure of individuals to tuberculosis and lifestyle factors are associated with the development of tuberculosis. This study aimed to quantify the relative effects of these factors at the individual (not geographical) level. For this, reason attempts were made to control for general socioeconoinic conditions (by matching for street-based postcodes) and ethnic origin (by matching on surname) in order to elucidate subtler lifestyle risk factors.

\section{Methods}

Subjects and procedure

A retrospective case-control questionnaire survey was conducted in the city of Liverpool in North-West 
England. Cases were defined as those notified with tuberculosis between January 1, 1989-June 30, 1996 to the Consultants for Communicable Disease Control for Liverpool and South Sefton Authorities (which form part of Liverpool City). The diagnosis was confirmed by examination of hospital case notes and bacteriological culture records. Records of all mycobactenal isolates were obtained frorn the four microbiology laboratories within Liverpool and patients with nontuberculous mycobacteria were excluded. Patients that were culture negative and not thought to have tuberculosis on clinical grounds were excluded. The case-definition criteria were either proven by culture or with an abnormal radiograph (or acid-fast bacilli (AFB) and granuloma and caseation) and positive tuberculin test and response to treatment.

Controls were recruited from the Liverpool and Sefton General Practitioner databases and matched to cases according to street-based postcode (full outcode plus first digit of incode), sex, date of birth \pm 3 yrs and ethnic origin [21].

\section{Recruitment}

Letters requesting participation in the study were sent, with a prepaid envelope and reply slip, to all cases notified between 1989-1996 (except if they were known to have died). All nonresponders (to a second letter 2 months later) were traced through the Office of National Statistics (ONS) to determine whether they were still alive and still resident in Liverpool (if not, they were excluded). Similar letters, also requesting consent to a tuberculin (Heaf) test, were sent to five controls matched to each case that had agreed to take part in the survey. If after 2 months no controls had agreed to take part, a further five controls were randomly selected from the matched list. Five controls per case were selected in an attempt to ensure that there were sufficient respondents.

\section{Interviews and Heaf testing}

A trained tuberculoslis specialist or respiratory research nurses visited participants in their homes between May 1995-March 1997 to complete the questionnaires. Controls were tuberculin tested by the Heaf method after the interviews had been conducted, to exclude current and/or previous tuberculosis infection. The skin tests were performed and read $(\sim 1$ week later) by the tuberculosis specialist nurse.

\section{The questionnaire}

The structured questionnaire was designed to assess: demographical variables, general living conditions (e.g. persons per household, migration, car ownership), access to luxury items (e.g. microwave, mobile phone, satellite television), personal, family and household education and employment history, diet, drinking and smoking habits, exercise, social and outside activities and medical history. To assess changes in circumstances over time, questions were related to the time of interview and to $2 \mathrm{yrs}$ before the diagnosis of tuberculosis. Controls were asked to answer questions relating to the same time periods as the cases to which they were matched.

\section{Statistical analyses}

Due to the small number of ethnic minority cases and controls, participants were classified as White and non-White (as self-ascribed). All occupations were recoded according to the Registrar General's Classification of Occupations (1980), and all housewives aged $>60 \mathrm{yrs}$, were classified as retired [22]. The highest household occupation (i.e. highest income) of either parent was used in the analysis of parental socioeconomic status.

Statistical analyses were performed for two time periods: 2 yrs before tuberculosis was diagnosed and the present day (i.e. when the interview was conducted). The time between interview and tuberculosis diagnosis ranged from $\sim 1$ month-7 yrs. Univariate analysis was carried out to assess the basic differences between cases and controls at both time periods. Analyses were also carried out, within cases and controls, to determine changes in lifestyle over time. Multiple logistic regression was used to identify confounding factors and measure the independent effects of each variable. This analysis was stratified according to the matching criteria by including sex, age, socioeconomic index and ethnicity in the model. All the statistical analyses were carried out using SPSS [23].

\section{Results}

\section{Recruitment}

A total of 430 individuals were identified $(61 \%$ of whom were culture positive) with tuberculosis during the 7-yr study period. Of these, $111(26 \%)$ had died and 110 were untraceable or had uncertain diagnosis. Of the 219 remaining cases, 144 replied $(66 \%)$ and 112 were interviewed $(61 \%$ culture positive). There were no significant differences between the age, sex or area of residence between all individuals notified and the cases recruited to the study.

A total of 198 suitable controls were recruited and 168 of these received a Heaf test. Those with a grade 3 or 4 response $(n=7)$ were interviewed and radiography performed to exclude active tuberculosis.

\section{General characteristics: matching of controls}

There was a good overall match between the average characteristics of both groups (table 1) with no significant difference between the sex distribution or age ranges: only 11 of 112 cases $(10 \%)$ were aged $<20$ yrs and $29 \%$ were $>65$ yrs.

There was a significantly lower proportion of non-Whites in the control group than in the cases. 
Table 1.-General characteristics of tuberculosis cases and controls (factors which could not be measured over time)

\begin{tabular}{|c|c|c|c|}
\hline Factors & $\begin{array}{c}\text { Cases } \\
\%\end{array}$ & $\begin{array}{c}\text { Controls } \\
\%\end{array}$ & p-Value \\
\hline Subjects $\mathrm{n}$ & 112 & 198 & \\
\hline \multicolumn{4}{|l|}{ Demographical } \\
\hline Male & 55.4 & 54.5 & \\
\hline Aged $>55$ yrs & 47.3 & 46.5 & \\
\hline Nonwhite & 14.3 & 6.6 & * \\
\hline Born in the UK & 86.6 & 96.0 & \# \\
\hline \multicolumn{4}{|l|}{ Housing/socioeconomic } \\
\hline Ever lived abroad & 32.1 & 20.7 & * \\
\hline Currently not working & 26.9 & 23.3 & \\
\hline Currently retired & 37.4 & 39.2 & \\
\hline $\begin{array}{l}\text { Highest household } \\
\text { occupation I/II }\end{array}$ & 74.0 & 81.0 & \\
\hline $\begin{array}{l}\text { Highest household } \\
\text { occupation III/IV }\end{array}$ & 19.6 & 15.6 & \\
\hline $\begin{array}{c}\text { Not taken holidays } \\
\text { abroad in } 10 \text { yrs }\end{array}$ & 48.1 & 35.2 & $*$ \\
\hline \multicolumn{4}{|l|}{ Lifestyle } \\
\hline Eat dairy products weekly & 88.4 & 96.5 & ** \\
\hline Eat meats/fish weekly & 94.6 & 96.0 & \\
\hline Eat salads/veg weekly & 95.5 & 99.5 & * \\
\hline Smoked for at least $30 \mathrm{yrs}$ & 46.4 & 34.0 & * \\
\hline \multicolumn{4}{|l|}{ Medical } \\
\hline Suffer from diabetes & 7.1 & 5.1 & \\
\hline Suffer from high blood pressure & 10.7 & 19.4 & * \\
\hline Had a gastrectomy & 1.9 & 1.0 & \\
\hline Taken gastric-acid inhibitors & 17.0 & 10.6 & \# \\
\hline Previously had BCG & 28.6 & 38.0 & \\
\hline Lived with anyone with $\mathrm{TB}$ & 31.5 & 11.3 & $* * *$ \\
\hline
\end{tabular}

BCG: bacille Calmette-Guérin vaccine; TB: tuberculosis. Chi-squared cases versus controls: ${ }^{*}: \mathrm{p}<0.05$; ${ }^{* *}$ : $\mathrm{p}<0.01$; ***: $\mathrm{p}<0.001 ;{ }^{*}: \mathrm{p}<0.005$.
This was due to difficulties in obtaining enough controls from ethnic minorities because of the low numbers in the Liverpool population: 3.8\% nonWhite (ONS 1991 Census Local Area Statistics). As a consequence, fewer cases were born in the UK and more had lived abroad (table 1). However, this difference in ethnic mix was controlled for in the multivariate analyses. Within the non-White group there was no significant (Chi-squared $=0.235, \mathrm{p}=0.89$ ) difference between the ethnicity of cases and controls: $35 \%$ were Black African; 24\% were from the Indian Subcontinent (Indian, Bangladeshi or Pakistani); and $41 \%$ were other Black or Asian (Chinese, Indonesian, Vietnamese, Malaysian, Arabic, Caribbean or Black British).

\section{Socioeconomic features of cases and controls}

The matching of socioeconomic status was reflected in the similarity in housing status and employment and education history of the cohorts. Thus, basic housing data (number of residents, number of bedrooms, overcrowding, type of heating, or damp in the house) did not differ between cases and controls at either time period. There was no significant difference in the current working status between cohorts (table 1): overall, 27\% were working; 17\% not working; $7 \%$ housewives; $10 \%$ in education and $39 \%$ retired. Also, the highest occupational position of the household did not differ between cases and controls (table 1) nor did the possession of most luxury items (access to a car/van, double glazing, house alarm, telephone, satellite dish or dishwasher) (table 2).

Table 2. - Univariate analysis of lifestyle and socioeconomic factors in tuberculosis (TB) cases compared with controls at different time periods

\begin{tabular}{|c|c|c|c|c|}
\hline Factors & Cases $^{\#}$ & Controls ${ }^{\#}$ & 2 years pre-TB & At interview \\
\hline \multicolumn{5}{|l|}{ Housing/socioeconomic } \\
\hline House owned/mortgaged & $0.83(0.24-2.88)$ & $1.1(0.59-1.86)$ & $0.65(0.4-1.01)^{*}$ & $0.65(0.41-1.04)^{*}$ \\
\hline More than one bathroom & $1.1(0.47-2.4)$ & $1.1(0.65-1.7)$ & $3.38(1.26-9.04)^{* *}$ & $2.67(1.1-6.7)^{*}$ \\
\hline Average income $>£ 10,000$ & $1.0(0.54-1.85)$ & $1.0(0.65-1.55)$ & $0.45(0.23-0.89)^{*}$ & $0.46(0.23-0.88)^{*}$ \\
\hline Working & $0.76(0.4-1.44)$ & $0.68(0.43-1.05)^{*}$ & $0.72(0.42-1.21)$ & $0.81(0.46-1.42)$ \\
\hline \multicolumn{5}{|l|}{ Luxury items in household } \\
\hline TV & $1.54(0.42-5.62)$ & $0.67(0.11-4.03)$ & $0.18(0.04-0.91)^{*}$ & $0.42(0.09-1.9)$ \\
\hline Video & $1.14(0.64-2.03)$ & $1.16(0.7-1.91)$ & $0.56(0.33-0.96)^{*}$ & $0.55(0.32-0.96)^{*}$ \\
\hline Satellite & $2.23(1.18-4.21)^{* *}$ & $2.08(1.27-3.4)^{\#}$ & $1.08(0.58-2.02)$ & $1.16(0.7-1.92)$ \\
\hline Microwave & $1.5(0.85-2.53)$ & $1.59(1.04-2.42) *$ & $0.85(0.53-1.37)$ & $0.79(0.48-1.3)$ \\
\hline Washing machine & $1.01(0.47-2.13)$ & $1.01(0.48-2.14)$ & $0.50(0.23-1.04)^{*}$ & $0.49(2.33-1.04)^{*}$ \\
\hline Compact disc player & $1.44(0.82-2.52)$ & $1.82(1.21-2.72)^{*}$ & $0.70(0.43-1.57)$ & $0.56(0.35-0.89)^{*}$ \\
\hline \multicolumn{5}{|l|}{ Lifestyle } \\
\hline Vitamins taken regularly & $1.86(0.95-3.64)^{*}$ & $1.38(0.89-2.14)$ & $0.53(0.29-0.98)^{*}$ & $0.71(0.42-1.2)$ \\
\hline At least $1 \mathrm{~h}$ exercise $\cdot$ day $^{-1}$ & $0.71(0.42-1.22)$ & $0.78(0.52-1.16)$ & $0.71(0.44-1.12)$ & $0.64(0.4-1.04)^{*}$ \\
\hline At least $1 \mathrm{~h}$ outside $\cdot$ day $^{-1}$ & $0.3(0.17-0.52)^{* * *}$ & $0.67(0.45-1.0)^{*}$ & $0.65(0.4-1.04)$ & $0.29(0.17-0.47)^{* * *}$ \\
\hline At least 5 days out $\cdot$ week $^{-1}$ & $0.55(0.28-1.06)^{*}$ & $0.75(0.43-1.32)$ & $0.76(0.39-1.45)$ & $0.55(0.31-0.97)^{*}$ \\
\hline Smoker & $0.61(0.34-1.06)^{*}$ & $0.95(0.6-1.51)$ & $2.33(1.4-3.88)^{* * *}$ & $1.46(0.87-2.47)$ \\
\hline Smoke at least 20 a day & $0.55(0.32-0.95)^{* *}$ & $0.88(0.57-1.37)$ & $2.39(1.48-3.86)^{* *}$ & $1.5(0.91-2.47)$ \\
\hline Drinker & $0.69(0.4-1.22)$ & $1.17(0.75-1.82)$ & $1.01(0.67-1.7)$ & $1.71(1.04-2.82)^{*}$ \\
\hline Drink $>30$ units $\cdot$ week $^{-1}$ & $0.46(0.19-1.03) *$ & $0.61(0.3-1.23)$ & $1.33(0.67-2.66)$ & $1.01(0.41-2.49)$ \\
\hline
\end{tabular}

\#: odds ratio (OR) and 95\% confidence interval (CI) for cases and controls 2 yrs prediagnosis versus interview and the significance associated with a change over time; 9 : OR, $95 \%$ CI and significance for differences between cases and controls at each time period. Cases: $\mathrm{n}=112$; controls: $\mathrm{n}=198$. Chi-squared: ${ }^{*}: \mathrm{p}<0.05 ;{ }^{* *}: \mathrm{p}<0.01 ;{ }^{* * *}: \mathrm{p}<0.001 ;{ }^{\#}: \mathrm{p}<0.005$. 
Some socioeconomic variables did differ between cases and controls. A lower proportion of cases owned or mortgaged their house, cases were significantly less likely to have more than one bathroom, and a lower proportion of cases earned $>£ 10,000$ per annum (table 1). In addition. cases were less likely to have a video or compact disc player or access to a washing machine.

\section{Lifestyle features of cases and controls}

Lifestyle variables that were measured at the time of interview only are shown in table 1 . Only 3\% of cases or controls were vegetarians, but cases ate dairy products (milk, cheese and yoghurt) and salads and vegetables significantly less frequently than controls. The majority of both cases and controls (70\%) stated that their diet had not changed in the last few years (Chi-squared $=0.06, \mathrm{p}=0.81$ ).

Despite the fact that cases were significantly less likely to have taken holidays in the last $15 \mathrm{yrs}$, foreign-born cases were more likely to have visited their country of birth than foreign-born controls: $53 \%$ compared with $25 \%$. Also, cases were significantly more likely to have had visitors from a non-British country of birth: $5.4 \%$ of cases had $>3$ visitors (up to 18 ) in the preceding 15 yrs.

A significantly higher proportion of cases had smoked cigarettes at some time in their life: $71 \%$ compared with $62 \%$ of controls (Chi-squared $=3.03$, $\mathrm{p}<0.05)$ and a higher proportion had smoked for $>30$ yrs (table 1). However, the age smoking started (mean $17 \mathrm{yrs}$, range $7-40, \mathrm{t}=1.14, \mathrm{p}=0.26$ ) did not differ between cases and controls.

There was also no difference in the occurrence of most long-term illnesses: overall $6 \%$ had diabetes; $12 \%$ heart disease; $4 \%$ a stroke; $43 \%$ arthritis or bone/joint problems; 3\% kidney disease; 5\% cancer; and $1.3 \%$ had had a gastrectomy. However, cases were significantly less likely to have high blood pressure (11\% compared with $19 \%$ ) and more likely to have taken gastric-acid inhibitors for $\geqslant 6$ months $(17 \%$ compared with $11 \%$ ). Also, cases were more likely to have lived with someone who had had tuberculosis (32\% compared with $11 \%$ ).

\section{Changes over time}

Variables that were recorded both 2 yrs before diagnosis and at the time of interview are shown in table 2. Some factors changed over time, but showed no significant difference between cases and controls: the proportion in work and having a satellite dish or microwave. Some factors were significantly different between cases and controls at both time periods but did not change over time: the proportion of owned or mortgaged houses, number of bathrooms, earning $>£ 10,000$ per annum and having a video or washing machine. Other factors differed between cases and controls either 2 yrs before diagnosis or at interview: having a television or compact disc player, taking vitamin supplements, taking exercise or going, out of the house and level of smoking and drinking. The most significant changes were that, compared to 2 yrs before diagnosis, cases were less likely to go out of the house and had reduced cigarette consumption (or given up smoking).

\section{Risk factors for tuberculosis (multivariate analysis)}

Each factor that showed a statistically significant difference between cases and controls at any time period was incorporated into a logistic regression model. The final models showed that there were multiple factors significantly associated with developing tuberculosis (table 3 ).

Two years before the diagnosis of tuberculosis, cases were less likely to have been born in the UK, more likely to have lived with someone with tuberculosis and more likely to have had a visitor from their country of birth. Also, they were less likely to have more than one bathroom, more likely to have smoked for $>30 \mathrm{yrs}$ and less likely to eat dairy products every week or to have high blood pressure. In addition to these risk factors, at the time of interview, cases were also significantly less likely to go out of the home during an average week.

Table 3.-Multivariate analysis of lifestyle and socioeconomic factors in cases compared with controls

\begin{tabular}{lll}
\hline Factors & OR & $95 \%$ CI \\
\hline Exposure & & $1.4-11.0$ \\
$\quad$ More likely to have been born abroad & 4.0 & $1.4-40.9$ \\
$\quad$ More likely to have had visitors from country of birth & 7.4 & $2.0-7.2$ \\
$\quad$ More likely to have lived with someone with TB & 3.8 & $1.3-12.4$ \\
Socioeconomic & 4.0 & $1.2-4.2$ \\
$\quad$ Less likely to have more than one bathroom & 2.3 & $1.4-10.3$ \\
Lifestyle & 3.8 & $1.2-5.7$ \\
$\quad$ More likely to have smoked for at least 30 yrs & 2.6 & \\
Mesical likely to have eaten dairy products every week & & \\
$\quad$ Less likely to have high blood pressure &
\end{tabular}

Data show the odds ratio (OR) for cases and $95 \%$ confidence intervals (CI) of the statistically significant factors remaining in the model. Cases: $n=112$; controls: $n=198$; TB: tuberculosis. 


\section{Discussion}

This study has shown that the risk factors for tuberculosis are multifaceted. Exposure to tuberculosis and being born abroad were both associated with an approximately five-fold increase in the probability of developing tuberculosis. Socioeconomically, cases were less likely to have more than one bathroom. Lifestyle differences showed that cases were more likely to smoke and less likely to eat dairy products. The only medical factor to show a difference was that cases were less likely to have high blood pressure than controls.

Selecting controls on a street-based postcode (full outcode plus first digit of the incode), age and ethnic origin resulted in some difficulty in recruiting controls because of the low proportion of non-White ethnic groups in the general Liverpool population. Five controls per case were contacted in order to achieve an adequate number of controls, and finally recruited 1.76 controls per case. Also, given the older age of tuberculosis cases and the death of $26 \%$ of cases over the 7-yr period, there was a relatively low recruitment rate. However, the general characteristics of all the cases (age, sex and locality) did not differ from the recruited cases.

There was a good level of matching for all basic demographical details between cases and controls. Ethnic groups were included in all multivariate analyses to control for confounding effects of this variable. Also, some subtle housing measures reflecting individual socioeconomic status did differ, with cases less likely to own or mortgage their house or to have more than one bathroom. The single biggest risk factor for tuberculosis using multiple logistic regression was exposure to visitors from the country of birth. This would suggest that tuberculosis is, in part, brought into the country from abroad. Household exposure to tuberculosis and being born abroad carried an approximately equal risk for tuberculosis. After controlling for the greater probability of cases being born abroad than controls, within both cases born in the UK and abroad, there was a greater probability of having lived with someone with tuberculosis than in controls.

Univariate analysis showed that cases were less likely to own their own home and were less likely to have a higher income, and multiple logistic regression showed that cases were less likely to have more than one bathroom (table 2). Despite the fact that this study attempted to control for local socioeconomic variables by using street based controls, subtle differences between housing conditions within the same locality were still evident. The detailed socioeconomic differences were paralleled by the tendency for cases to have fewer luxury items such as videos and compact disc players and washing machines. However, cases were as likely to own satellite dishes, which may be related to the fact that they stayed at home more after their illness developed (see later).

Prior to diagnosis, cases were more likely to smoke and were heavier smokers than controls. Smoking is known to increase the risk of tuberculosis approximately three-fold [12]. There was no difference in the frequency or intensity of alcohol consumption 2 yrs before tuberculosis was diagnosed. At the time of interview, cases were less likely to eat dairy products or take multivitamins regularly. Milk, calcium and vitamin $\mathrm{D}$ were used in the treatment of tuberculosis before specific chemotherapy was available [24]. The results of this study are compatible with a protective effect of dairy products against tuberculosis [25]. These factors would suggest that cases generally had less healthy lifestyles than controls.

Gastrectomy is known to increase the risk of tuberculosis approximately five-fold [26]. Here, univariate analysis showed that cases were more likely than controls to have taken $\mathrm{H} 2$ gastric-acid inhibitors within the past 6 months (table 1), but this was not significant in multiple logistic regression. Nurnbers in the present study were possibly too low for an effect to be noticeable. Cases were less likely to have high blood pressure than controls. This might reflect a lifestyle associated with a high fat and dairy product intake in controls, which does not appear to occur in tuberculosis patients.

The structure of this study was such that it was possible to assess changes in lifestyle and socioeconomic factors that were associated with having tuberculosis. Cases reduced their smoking and alcohol consumption, whereas controls showed no such changes over the same time interval. Tuberculosis patients will have been given advice to stop smoking and reduce alcohol consumption at the turie of their diagnosis. It is interesting that this advice seems to have been adopted by a significant number of patients, who continued to live a healthier lifestyle at the time of interview (which may have been up to 7 yrs after the illness).

Cases had reduced the time spent outdoors after their illness to a level significantly less than the time spent outdoors by controls. The reasons for this are unclear, but it is possible that cases had a chronic disability which reduced their activity but which was not measured in the survey. Anecdotal evidence suggests that being diagnosed with tuberculosis reduced the social contacts made by the cases and that tuberculosis continues to be a stigmatizing diagnosis in White as well as immigrant communities.

A previous case-control study demonstrated that those who developed tuberculosis had experienced a concentration of social disturbances, such as domestic strife, residential and occupational changes, and personal crises during the 2 yrs preceding the development of tuberculosis [27]. The authors concluded that those who became ill did so in a situation of stress, which would be conducive to lowered resistance, and postulated psychological crisis as one precipitant cause. These factors were not examined specifically in this study, but no significant differences were found between marital status or recent change of housing in cases compared with controls. Unfortunately, the study did not involve specific measures of physical or mental wellbeing and future studies in this area would benefit from this approach.

Although the present study took place in a city with a predominantly White population, the expected effects of immigration and/or contact with 
tuberculosis were still observed. Also, the known association with smoking as a risk factor for tuberculosis was confirmed. After controlling for the economic status of the locality in which patients live, minor lifestyle differences compatible with less disposable income were observed in cases compared with controls. The protective effects of a dairy-rich diet have been observed, but the often-held view of the association with alcohol was not seen. Despite following a healthier lifestyle after diagnosis and treatment, cases appear to be more socially isolated. The reasons for this deserve more detailed exploration to distinguish the possible effects of chronic physical ill health from potential social stigmatization, which is inappropriate for the 21st century.

The results of this study provide evidence that increased global travel, particularly to countries of high prevalence, poses a significant risk of persons being exposed to, and consequently developing, tuberculosis. Trends in international travel mean that barriers between countries with and without high levels of tuberculosis are disappearing. Policies to improve awareness of the risk from tuberculosis related either to travel or to receiving visitors from these areas are now essential preventative measures. While smoking levels decreased in cases (postdiagnosis), diet was still poorer in cases than controls at the time of interview. The importance of diet has been identified elsewhere and should be emphasized in the clinical setting. Finally, the social wellbeing of cases may have decreased after diagnosis through reduced contact with friends and relatives. The stigma still attached to tuberculosis must be addressed through general information campaigns.

\footnotetext{
Acknowledgements. The authors are very grateful to North Mersey Community Trust and University Hospital Aintree for permitting the involvement of three tuberculosis/respiratory specialist nurses (S. Jamicson, K. Pye and D. Frost) in interviewing participants.
}

\section{References}

1. World Health Organisation. Global tuberculosis Programme. WHO report on the Tuberculosis Epidemic, 1997.

2. Hayward AC, Watson JM. Tuberculosis in England and Wales 1982-1993: notifications exceeded predictions. CDR Review 1995; 5: R29-R33.

3. Murray CJL, Styblo K, Rouillon A. Tuberculosis in developing countries: burden, intervention and cost. Bull Int Union Tuberc Lung Dis 1990; 65: 6-24.

4. De Cock KM. Tuberculosis control in resource-poor settings with high rates of HIV infection. Am J Public Health 1996; 86: 1071-1073.

5. Doherty MJ, Spence DPS, Davies PDO. The increase in tuberculosis notifications in England and Wales since 1987. Tuber Lung Dis 1995; 76: 196-200.

6. McKenna M, McCray E, Onorato I. The epidemiology of tuberculosis among foreign-born persons in the United States, 1986-1993. N Engl J Med 1995; 221: 1071-1076.
7. Kumar D, Watson JM, Charlett A, Nicholas S, Darbyshire JH, on behalf of a Public Health Laboratory Service/British Thoracic Society/Department of Health Collaborative Group. Tuberculosis in England and Wales in 1993: results of a national survey. Thorax 1997; 52: 1060-1067.

8. Spence DPS, Hotchkiss J, Williams CSD, Davies PDO. Tuberculosis and poverty. BMJ 1993; 307: 759-761.

9. Bhatti N, Law MR, Morris JK, Halliday R, Moore Gillon J. Increasing incidence of tuberculosis in England and Wales: a study of the likely causes. BMJ 1995; 310: 967-969.

10. Mangtani P, Jolley DJ, Watson JM, Rodrigues LC. Socioeconomic deprivation and notification rates for tuberculosis in London during 1982-1991. BMJ 1995; 310: 963.

11. Drucker E, Alcabes P, Bosworth W, Sckell B. Childhood tuberculosis in the Bronx, New York. Lancet 1994; 343: 1482-1485.

12. Alcaide J, Altet MN, Parron I, et al. Cigarette smoking as a risk factor for tuberculosis in young adults: a case-control study. Tuber Lung Dis 1996; 77: 112-116.

13. Bloom BR, Murray CJL. Tuberculosis: commentary on a re-emergent killer. Science-Wash 1992; 257: 1055-1064.

14. Kumar D, Liese J, Citron K, Watson J. Tuberculosis among the homeless in London. Tubercle 1995; 75: 73-74.

15. Davies PDO. The slowing of the decline in tuberculosis notifications and HIV infection. Resp Med 1989; 83: 321-322.

16. Powell KE, Farer LS. The rising age of the tuberculosis patient: a sign of success and failure. $J$ Infect Dis 1980; 142: 946-948.

17. Brudney K, Dobkin J. Resurgent tuberculosis in New York City. Human immunodeficiency virus, homelessness, and the decline of tuberculosis control programs. Am Rev Respir Dis 1991; 144: 745-749.

18. Friednian LN, Williams MT, Singh TP, et al. Tuberculosis, AIDS, and death among substance abusers on welfare in New York City. N Engl J Med 1996; 334: 828-833.

19. Tocque K, Doherty MJ, Bellis MA, Spence DPS, Williams CSD, Davies PDO. Tuberculosis notifications in England: The relative effects of poverty and ethnicity. Int J Tuberc Lung Dis 1998; 2: 213-218.

20. Rouillin A. The epidemiological basis of tuberculosis control. In: Davies PDO, ed. Clinical Tuberculosis. London, Chapman and Hall, 1998; pp. 35-52.

21. Nisar N, Davies PDO. Experience of tuberculosis in South East Asian immigrants to Liverpool. Respir Med 1991; 85: 219-222.

22. Registrar general's classification of occupation. Occupational Classification Volume 1. London, Office of National Statistics, 1990.

23. Norusis MJ. SPSS for Windows. Chicago, USA, SPSS Inc., 1993.

24. Davies PDO. A possible link between vitamin D deficiency and impaired host defence to Mycobacterium tuberculosis. Tubercle 1985; 66: 301-306.

25. Finch PJ, Millard FJC, Maxwell JD. Risk of tuberculosis in immigrant Asians; culturally acquired immunodeficlency? Thorax 1991; 46: 1-5.

26. Reider HL, Cauthen GM, Comstock GW, Sneider DE. Epidemiology of tuberculosis in the United States. Epidemiol Review 1989; 11: 79-98.

27. Fineberg HV, Wilson ME. Social vulnerability and death by infection. $N$ Engl J Med 1996; 334: 859-860. 